\title{
a Coleman integral?
}

\section{Jennifer S. Balakrishnan}

\section{Introduction}

Robert Coleman wrote a series of papers in the 1980s [Col82, Col85b, CdS88] where he developed a theory of $p$-adic line integration on curves and higher-dimensional varieties with good reduction at $p$. He gave a number of spectacular applications for these integrals, thereby demonstrating that they are a powerful tool in arithmetic geometry. These integrals are now known as Coleman integrals. The theory has since been extended in a number of different directions by Berkovich, Besser, Colmez, Vologodsky, and Zarhin, among others. Moreover, these integrals are amenable to computation, and implementations in various contexts are available in the computer algebra systems SageMath and Magma.

We discuss how to construct and compute these integrals and conclude by mentioning a few applications. For ease of exposition, we will assume that $X$ is a smooth projective curve of genus $g$ defined over $\mathbf{Q}$. We will also assume that $p$ is a prime of good reduction for $X$, which means that locally, the equations defining $X$ can be written with coefficients in $\mathbf{Z}_{p}$, so that when we reduce these modulo $p$, we obtain a smooth curve over $\mathbf{F}_{p}$.

Suppose $\omega$ is a meromophic 1-form of the second kind on $X_{\mathrm{Q}_{p}}$, i.e., a 1 -form with residue zero at all poles. What does it mean to compute the line integral $\int_{P}^{Q} \omega$ for points $P, Q \in X\left(\mathbf{Q}_{p}\right)$ ? The crux of the matter is understanding a $p$-adic "path" between the points $P$ and $Q$, in particular if $P$ and $Q$ are $p$-adically far away from each other.

Jennifer S. Balakrishnan is a Clare Boothe Luce Assistant Professor in the department of mathematics and statistics at Boston University. Her email address is jbala@bu.edu.

Communicated by Notices Associate Editor Daniel Krashen.

For permission to reprint this article, please contact:

reprint-permission@ams .org.

DOI: https://doi.org/10.1090/noti1872

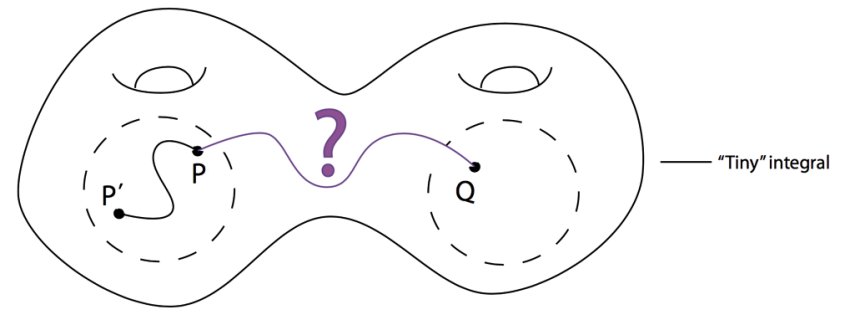

First we introduce a bit of terminology. Let $X^{a n}$ denote the rigid analytic space over $\mathbf{Q}_{p}$ associated to $X$ (this is an analogue of a complex analytic space over a nonarchimedean field). There is a natural specialization map from $X^{a n}$ to its special fibre $\bar{X}$ given by reduction $\bmod p$. The fibres of this map are open unit disks, called residue disks.

Now fix a residue disk $D$ and consider $P \in D$. We can compute a uniformizing parameter $t$ at $P$, and if $P^{\prime} \equiv P$ $(\bmod p)$, the "tiny integral" $\int_{P}^{P^{\prime}} \omega=\int_{0}^{t\left(P^{\prime}\right)} \omega(t) d t$, computed by a change of variables, converges. In particular, this is the Coleman integral of $\omega$ between $P$ and $P^{\prime}$.

Example 1. Let $X: y^{2}=x^{5}+2 x+1$ over $\mathbf{Q}_{5}$, and consider the points $P=(0,1), P^{\prime}=(5,56)$ and the differential form $\frac{d x}{2 y}$. We have that $t=x$ is a uniformizing parameter at $P$, and we compute $y=1+t-\frac{1}{2} t^{2}+\frac{1}{2} t^{3}-$ $\frac{5}{8} t^{4}+\frac{11}{8} t^{5}-\frac{29}{16} t^{6}+\frac{45}{16} t^{7}-\frac{589}{128} t^{8}+O\left(t^{9}\right)$. We have

$$
\begin{aligned}
& \int_{P}^{P^{\prime}} \frac{d x}{2 y}=\int_{0}^{5} \frac{d x(t)}{2 y(t)} d t \\
& \quad=3 \cdot 5+3 \cdot 5^{2}+2 \cdot 5^{3}+2 \cdot 5^{4}+3 \cdot 5^{5}+3 \cdot 5^{6}+O\left(5^{7}\right) .
\end{aligned}
$$

However, if $Q \not \equiv P(\bmod p)$, the strategy above does not work. Indeed, as open unit disks in the $p$-adic topology are either disjoint or identical, we seem to have a serious problem: we can compute tiny integrals within any 
given residue disk, but how do we carry out analytic continuation to ensure compatibility between different residue disks?

Coleman solved this problem using Dwork's principle of analytic continuation along Frobenius, that is to say, using the fact that these integrals are Frobenius equivariant. By computing the action of Frobenius on differentials, Coleman showed that there is a canonical path between two points, which allowed him to compute integrals between different disks. One works over a wide open subspace $V$ of $X^{a n}$ (obtained by removing from $X^{a n}$ a finite number of closed disks of radius less than 1) and uses Frobenius (a rigid analytic map) to write down a linear system. The key insight is that by (proofs of) the Weil conjectures, we know the possible eigenvalues of Frobenius on $p$-adic (MonskyWashnitzer or rigid) cohomology. We make this more precise below.

\section{Integrals Between Different Residue Disks}

First we record a number of useful properties of the Coleman integral:

Theorem 2 (Coleman). Let $\eta, \xi$ be 1-forms of the second kind on a wide open subspace $V$ of $X^{\text {an }}$ and $P, Q, R \in V$. The definite Coleman integral has the following properties:

1. Linearity: $\int_{P}^{Q}(a \eta+b \xi)=a \int_{P}^{Q} \eta+b \int_{P}^{Q} \xi$.

2. Additivity in endpoints: $\int_{P}^{Q} \xi=\int_{P}^{R} \xi+\int_{R}^{Q} \xi$.

3. Change of variables: If $V^{\prime} \subset X^{\prime}$ is a wide open subspace of a rigid analytic space $X^{\prime}$ and $\phi: V \rightarrow V^{\prime}$ a rigid analytic map then $\int_{P}^{Q} \phi^{*} \xi=\int_{\phi(P)}^{\phi(Q)} \xi$.

4. Fundamental theorem of calculus: $\int_{P}^{Q} d f=f(Q)-$ $f(P)$ for $f$ a rigid analytic function on $V$.

The rigid cohomology group $H_{\text {rig }}^{1}\left(X / \mathbf{Q}_{p}\right)$ is a $2 g$ dimensional $\mathbf{Q}_{p}$-vector space that is equipped with an action of $p$-power Frobenius $F_{p}$ that is lifted from the special fibre $\bar{X}$. By the work of Baldassarri and Chiarellotto, there is an isomorphism between rigid and algebraic de Rham cohomology. Let $\left\{\omega_{0}, \ldots, \omega_{2 g-1}\right\}$ be a basis of $H_{\text {rig }}^{1}\left(X / \mathbf{Q}_{p}\right)$.

One first computes the action of $p$-power Frobenius $F_{p}$ on each differential in the basis and reduces using relations in cohomology to obtain

$$
F_{p}^{*}\left(\omega_{i}\right)=d f_{i}+\sum_{j=0}^{2 g-1} \Phi_{i j} \omega_{j},
$$

where each $f_{i}$ is an element of a ring of overconvergent functions associated to $X_{\mathbf{Q}_{p}}$, and $\Phi \in M_{2 g \times 2 g}\left(\mathbf{Q}_{p}\right)$. In the case of hyperelliptic curves, the computation (1) is Kedlaya's algorithm, and in the case of smooth curves, this was recently made into a practical algorithm by Tuitman. Then one uses properties of the Coleman integral to compute the values of integrals on basis differentials between points
$P, Q$ where the $f_{i}$ converge, starting from

$$
\int_{F_{p}(P)}^{F_{p}(Q)} \omega_{i}=\int_{P}^{Q} F_{p}^{*} \omega_{i}
$$

and using (1) to deduce the following:

$$
\begin{aligned}
& \sum_{j=1}^{2 g}(\Phi-I)_{i j}\left(\int_{P}^{Q} \omega_{j}\right) \\
& \quad=f_{i}(P)-f_{i}(Q)-\int_{P}^{F_{p}(P)} \omega_{i}-\int_{F_{p}(Q)}^{Q} \omega_{i} .
\end{aligned}
$$

In particular, since the eigenvalues of the matrix $\Phi$ are algebraic numbers of complex absolute value $p^{1 / 2}$, the matrix $\Phi-I$ is invertible, and we obtain the integrals of basis differentials between $P$ and $Q$.

Example 3. As in our previous example, let $X: y^{2}=x^{5}+$ $2 x+1$ over $\mathbf{Q}_{5}$. Now consider the points $P=(0,1), Q=$ $(1,2)$. Using $(2)$, we compute in SageMath that

$\int_{P}^{Q} \frac{d x}{2 y}=2 \cdot 5+4 \cdot 5^{2}+3 \cdot 5^{3}+4 \cdot 5^{4}+4 \cdot 5^{5}+2 \cdot 5^{6}+O\left(5^{7}\right)$.

Remark 4. One can instead compute the Coleman integrals of regular 1-forms by passing to the Jacobian of the curve and rescaling so that the endpoints of integration are in the residue disk of the identity. Pulling back to the curve, this allows one to rewrite any given Coleman integral as a sum of tiny integrals. The main advantage of the approach with Frobenius is that it generalizes easily to iterated Coleman integrals.

Coleman and de Shalit, as well as Besser [Bes02], defined iterated Coleman integrals

$$
\int_{P}^{Q} \xi_{n} \cdots \xi_{1}
$$

which behave formally like iterated path integrals

$$
\int_{0}^{1} \int_{0}^{t_{1}} \cdots \int_{0}^{t_{n-1}} f_{n}\left(t_{n}\right) \cdots f_{1}\left(t_{1}\right) d t_{n} \cdots d t_{1} .
$$

Besser and de Jeu were the first to give an algorithm to compute iterated Coleman integrals, in the case of $X=$ $\mathbf{P}^{1} \backslash\{0,1, \infty\}$. These integrals are defined by the $p$-adic differential equations

$$
\begin{cases}\operatorname{Li}_{0}(z) & =\frac{z}{1-z} \\ d \mathrm{Li}_{n+1}(z) & =\mathrm{Li}_{n}(z) \frac{d z}{z}, n \geq 0\end{cases}
$$

and are $p$-adic polylogarithms, which via a $p$-adic analogue of Beilinson's conjecture, are conjecturally related to special values of $p$-adic $L$-functions. In the case of higher genus curves, one can compute $n$-fold iterated Coleman integrals by applying (1) to each differential in the integrand, then relating $n$-fold integrals to $(n-1)$-fold integrals and making an observation about the eigenvalues of the matrix $\Phi^{\otimes n}$, to produce the appropriate analogue of (2). 


\section{Applications}

Coleman and Gross described how to use Coleman integrals to compute $p$-adic heights on Jacobians of curves, which allows one to compute interesting arithmetic invariants, such as $p$-adic regulators. Indeed, there is a (global) $p$-adic height pairing on the Jacobian of a curve that, in many ways, parallels the story of the canonical NéronTate height: the Coleman-Gross $p$-adic height is a bilinear form $h$ that can be computed via a decomposition

$$
h\left(P_{1}, P_{2}\right)=\sum_{v} h_{v}\left(P_{1}, P_{2}\right)
$$

into local heights $h_{v}$. While the local heights at primes $v \neq p$ are computed via arithmetic intersection theory (just as in the Néron-Tate height, though the intersection multiplicities are weighted with $p$-adic logarithms), the local height pairing at the prime $v=p$ is given in terms of a Coleman integral. By computing global $p$-adic heights on a basis of the Mordell-Weil group of the Jacobian, one can calculate a $p$-adic regulator, which is one of the invariants appearing in a $p$-adic analogue of the Birch and Swinnerton-Dyer conjecture.

Coleman also used these integrals to compute torsion points on Jacobians. He further gave a beautiful reinterpretation [Col85a], via his eponymous integrals, of the classical method of Chabauty for showing finiteness of the number of rational points on curves $X / \mathbf{Q}$ whose Jacobians have rank less than their genus. On each residue disk, the Coleman integrals are locally analytic and can be written as convergent $p$-adic power series, with finitely many zeros. By bounding the number of zeros of these integrals (where this zero locus, which we denote as $X\left(\mathbf{Q}_{p}\right)_{1}$, is a finite set of $p$-adic points containing the set of rational points), he gave an upper bound on the number of rational points on such curves. The Chabauty-Coleman method has been generalized in a number of ways, for instance, by removing the hypothesis that $p$ must be a prime of good reduction. This variant was used by Stoll and KatzRabinoff-Zureick-Brown to give a uniform bound on the number of rational points on curves whose Jacobians have rank at most $g-3$.

Iterated Coleman integrals are conjectured to satisfy striking relationships when evaluated on rational points on curves, with no restriction on the rank of the Jacobian of the curve. Kim's construction of Selmer varieties [Kim09] gives a sequence of sets

$$
X\left(\mathbf{Q}_{p}\right)_{1} \supset X\left(\mathbf{Q}_{p}\right)_{2} \supset \cdots \supset X\left(\mathbf{Q}_{p}\right)_{n},
$$

where each set $X\left(\mathbf{Q}_{p}\right)_{k}$ is described by $k$-fold iterated Coleman integrals and contains the set of rational points $X(\mathbf{Q})$. One would like to show that, for a given curve $X / \mathbf{Q}$, there is a computable depth $\ell$ at which the set $X\left(\mathbf{Q}_{p}\right)_{\ell}$ is finite. Moreover, one would like to compute the set $X\left(\mathbf{Q}_{p}\right)_{\ell}$. Some recent progress has been made on understanding quadratic Chabauty-i.e., studying the set $X\left(\mathbf{Q}_{p}\right)_{2}$-by constructing integrals that vanish on the set of points $X\left(\mathbf{Q}_{p}\right)_{2}$ for curves with extra structure and Mordell-Weil rank equal to $g$. More generally, by carrying out Kim's nonabelian Chabauty program, one hopes to discover new relationships among iterated Coleman integrals, thereby leading to a new, constructive proof of finiteness of rational points on higher genus curves.

\section{References}

[Bes02] Besser A. Coleman integration using the Tannakian formalism, Math. Ann. 322 (2002), 19-48. MR1883387

[CdS88] Coleman RF, de Shalit E. $p$-adic regulators on curves and special values of $p$-adic $L$-functions, Invent. Math. 93 (1988), no. 2, 239-266. MR948100

[Col82] Coleman RF. Dilogarithms, regulators and $p$-adic $L$-functions, Invent. Math.69 (1982), no. 2, 171-208. MR674400

[Col85a] Coleman RF. Effective Chabauty, Duke Math. J. 52 (1985), no. 3, 765-770. MR808103

[Col85b] Coleman RF. Torsion points on curves and $p$-adic abelian integrals, Ann. of Math. (2) 121 (1985), no. 1, 111168. MR782557

[Kim09] Kim M. The unipotent Albanese map and Selmer varieties for curves, Publ. Res. Inst. Math. Sci. 45 (2009), no. 1, 89-133. MR2512779

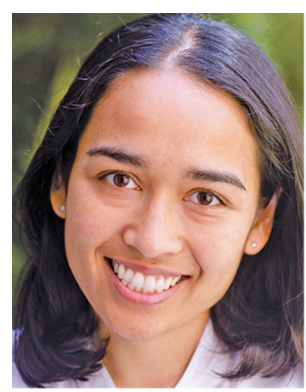

Jennifer S.

Balakrishnan

Credits

Photo of Jennifer S. Balakrishnan is courtesy of the author. 